Inwieweit unsere Ansichten, die zunächst nur als Arbeitshypothesen gelten sollen, eine Klärung der bisher theoretisch dunklen Phototropieerscheinungen berbeiführen werden, muß durch weitere Untersuchungen auf möglichst breiter Basis dargetan werden. Nur soviel soll bier noch betont werden, daB die phototropen Zustandsänderungen der Stilbenderivate grundsätzlich verschieden sind von denen der Hydrazone, Fulgide usw. Die Stilbenderivate nehmen also eine Sonderstellung unter den phototropen Stoffen ein.

Hrn. Kurt Thormann sagen wir für seine bei einem Teile der Arbeit geleistete Hilfe unseren besten Dank.

Leipzig, Chem. Universitätslaboratorium.

Leverkusen a. Rh. und Breslau.

161. A. Sieverts und H. Oehme: Die Dampfdrucke flüssiger Zinn-amalgame mit niedrigen Quecksllbergehalten.

[Mitteilung aus d. Laboratorium f. angewandte Chemie d. Universităt Leipzig.]

(Eingegangen am 26. Mărz 1913.)

Für die Lösungen der Gase in festen und flüssigen Metallen gilt im allgemeinen die Regel, daß bei gegebener Temperatur die gelöste Menge $m$ der Quadratwurzel aus dem Gasdruck $p$ proportional ist $\left(m=x p^{2 / s}\right)$. Die Beziehung läßt sich für die $z$ weiatomigen Gase $\left(\mathrm{H}_{2}, \mathrm{O}_{2}, \mathrm{~N}_{2}\right)$ unter der Voraussetzung ableiten, daß jedes Gasmolekül beim Übergang in die Lösung in zwei Teile, d. h. in die Atome zerfältt. Bei einatomigen Gasen ist ein solcher Zerfall nicht denkbar. Für sie darf also auch die Quadratwurzel-Beziehung nicht gelten, die ja eine Spaltung der Gasmoleküle voraussetzt. Vielmehr läßt sich auf Grund des Verteilungssatzes vorher sagen, da $\beta$ die gelöste Menge m der n-ten Potenz des Gasdrucks proportional sein $m u B\left(m=* p^{n}\right)$, wenn $n$ die Anzahl der Atome in jedem gelösten Molekül bedeutet. Sind auch die gelösten Moleküle einatomig, so wird $n=1$ und $m=x p$, d. h. es gilt das $H$ enrysche Gesetz. In allen anderen Fällen muß n größer als eins sein $\left.{ }^{2}\right)$.

Um diese Verhältnisse experimentell zu prüfen, haben Sieverts und Bergner ${ }^{3}$ ) vor kurzem versucht, die Löslichkeit der einato-

3) Vergl. z. B. Sieverts, Z. El. Ch. 16, 707 [1910].

ग) Bei teilweiser Assoziation ergeben sich weniger einfache Formeln, die sich aus den Gleichgewichtsbedingungen in der Lösung ableiten Iassen.

3) B. 45, 2576 [1912]. 
migen Gase Helium und Argon in festen und flüssigen Metallen festzustellen. Trotzdem zahlreiche Metalle geprüft wurden, gelang es in keinem Falle, eine sicher meßbare Absorption nachzuweisen. Wir haben deshalb ein einatomiges $G$ as gewählt, dessen Löslichkeit in Metallen sicher bekannt ist, nämlich den Quecksilberdampf. Als lösendes Metall diente $\mathrm{Zinn}$, weil es leicht schmelzbar ist, praktisch keinen Dampfdruck bat und mit Quecksilber keine chemischen Verbindungen bildet.

\section{A usführung der Versuche:}

Erstes Verfahren: Bei den ersten Versuchen haben wir ein Quarzmanometer verwendet. Das Quarzkölbchen zur Aufnahme des Amalgams hatte einen Inhalt von $14.5 \mathrm{ccm}$, die übrigen Teile des von Heräus gelieferten Quarzapparates entsprachen den Angaben von Bodenstein ${ }^{1}$ ). Bei den Druckmessungen hatten wir mit den Schwierigkeiten zu kämpien, die bei Tensionsbestimmungen von Lösungen immer aufzutreten pflegen. Die Einstellungen waren beim Erhitzen und Abküblen nur schlecht reversibel, offenbar, weil beim Verdampfen und Wiederauflösen des Quecksilbers die Konzentration an der AmalgamOberfläche schwankte und sich nur langsam durch Diffusion ausglich. Ein Durchschütteln der Lösung war unmöglich, denn der verwendete Apparat darf nicht im geringsten erschüttert werden. Wir haben deshalb nach diesem Verfahren nur zwei Versuchsreihen durchgeführt. Von den Messungen seien nur die bei hohen Temperaturen und größeren Drucken ausgeführten mitgeteilt, weil sie allein hinreichend reproduzierbar waren.

Tabelle I.

\begin{tabular}{|c|c|c|c|}
\hline \multicolumn{2}{|c|}{$\begin{array}{c}\text { Amalgam A } \\
97.8 \% \mathrm{Sn} \quad 2.2 \% \mathrm{Hg}\end{array}$} & \multicolumn{2}{|c|}{$\begin{array}{c}\text { Amsigam B } \\
90 \% \mathrm{Sn} \\
10 \% \mathrm{Hg}\end{array}$} \\
\hline Temperatur & $\begin{array}{c}\text { Druck } \\
\mathrm{mm} \mathrm{Hg}\end{array}$ & Temperatur & $\begin{array}{c}\text { Druck } \\
\mathrm{mm} \mathrm{Hg}\end{array}$ \\
\hline $\begin{array}{l}515 \\
528^{*} \\
619 \\
720\end{array}$ & $\begin{array}{l}173 \\
190^{*} \\
349 \\
634\end{array}$ & $\begin{array}{l}427 \\
477 \\
503 \\
515 \\
528\end{array}$ & 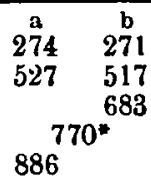 \\
\hline
\end{tabular}

Die mit ${ }^{-}$bezeichneten Zahlen sind intrapoliert. Die Ergebnisse werden am Schluß im Zusammenbang mit den übrigen Messungen besprochen werden.

1) Z. El. Ch. 16, 961 [1910]. 
Zweites Verfahren: Die Drucke des Amalgams wurden in einem evakuierten Manometer durch die Höhe einer Säule von flüssigem Zinn gemessen. Das nach dem Blasen sorgfältig gekühlte Manometer hatte die aus Fig. 1 ersichtliche Form '). Seine Länge betrug etwa $30 \mathrm{~cm}$, das weitere Glasrohr hatte $8 \mathrm{~mm}$, die Capillare $1.2 \mathrm{~mm}$ lichte Weite. Unterhalb der Ansatzstelle an dem weiten Schenkelrohr war die Capillare scharf nach unten gebogen, um das Übertreten von Quecksilberdampfblasen in die Capillare zu verbüten. Eine kleine Erweiterung der Capillare diente als Zinnreservoir und verbinderte ein Übertreten von Amalgam in die Capillare. Damit nicht ein Ab. reißen des Zinnfadens auftrat, mußte die Erweiterung möglichst gut konisch in die Capillare ein- und auslaufeo.

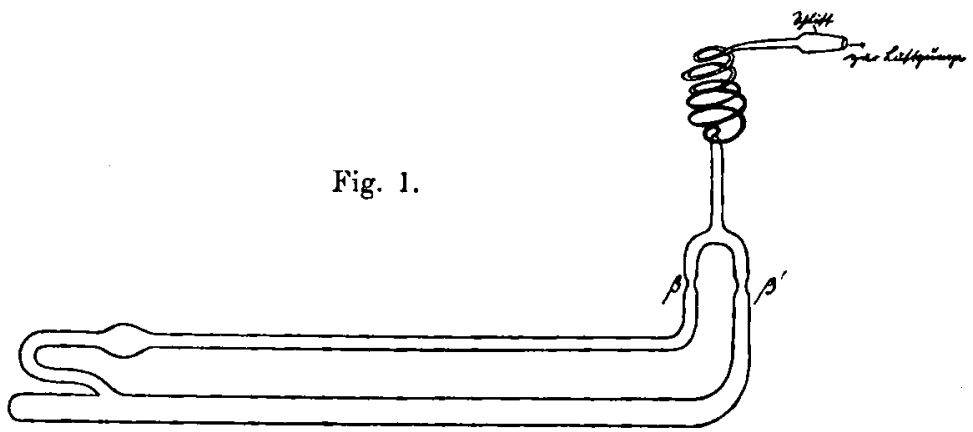

Das mit Bichromat-Schwefelsäure-Mischung gut gereinigte und sorgfältig getrocknete Manometer wurde mit etwa $25 \mathrm{~g}$ Zinn $\gg \mathrm{Kabl}$ baum* beschickt und an ein Y-Rohr angeschmolzen, das durch eine Kundtsche Feder und einen Glasschliff zur Quecksilberpumpe führte. Unter das horizontal liegende Manometer (Fig. 1) wurde nun eine eiserne Wanne mit dem festen eutektischen Gemisch von Kalium- und Natriumnitrat geschoben, langsam erwärmt, und zugleich die Pumpe in Tätigkeit gesetzt. Scbließlich gelangte der Apparat in das flïssig gewordene, etwa $300^{\circ}$ warme Salpeterbad. Nachdem die letzten Gasreste aus dem geschmolzenen $\mathrm{Zinn}$ entfernt waren, wurde das Manometer bei $\beta$ und $\beta^{\prime}$ abgeschmolzen, schnell aufgerichtet, um das flüssige Metall auch in den capillaren Schenkel zu bringen, und in einer Hülle von Asbestpapier abgekühlt. Der erkaltete Apparat wurde geöffnet, in den weiten Schenkel eine gewogene.Menge reinsten Quecksilbers eingefüllt, und das so fertig beschickte Manometer nocbmals in derselben Weise wie rorber mit der Luftpumpe verbunden, in der Kälte leer gepumpt und abgeschmolzen. Endlich wurde das weite Schenkel-

1) Der Ansatz oberhalb $\beta, \beta^{\prime}$ war zunächst nicht vorhanden. 
rohr in $2 \mathrm{~cm} \Lambda$ bstand von der Metalloberfläche an einer vorher angebrachten Verengung abgeschmolzen. Das Manometer hatte jetzt die in Fig. 2 gezeichnete Form (ohne Skala). Der capillare Schenkel wurde nun mit einer Drahtschlinge gefaßt, das weite kurze Rohr allein in das heiße Salpeterbad getaucht, und durch Hin- und Herschwenken das Amalgam gut durchgemischt, während das Zinn in der horizontal über dem Bad liegenden Capillare fest blieb. Beim Herausnebmen wurde der Apparal so aufgerichtet, daß $z$ wischen dem flüssigen Amalgam und dem Zinnfaden kein leerer Raum blieb. Nach dem Erkalten wurde eine in Millimeter geteilte, mit Platinschwarz durch Einbrennen gefärbte Milchglasskala an das Capillarrobr angeschmolzen (Fig. 2). Die Drucke waren auf diese Weise an der Skala direkt in $\gg m$ Zinn* ablesbar; die geringen Differenzen in den spezifiscben Gewichten der Amalgame und des Zinns bonnten vernachlässigt werden, auch eine Korrektur für die Capillarwirkung des langen Manometerschenkels erwies sich als überflüssig.

A usführung der Versuche: Als Heizbad diente ein unten balbkugelig geschlossener Glaszylinder aus Jenaer Hartglas von $30 \mathrm{~cm}$ Höhe und $4.5 \mathrm{~cm}$ Durchmesser, der mit einem gläsernen Luftmantel umgeben war. In den Zylinder wurden zunächst große Stücke einer erstarrten Schmelze des Eutektikums $\left(\mathrm{NaNO}_{2}+\mathrm{KNO}_{3}\right)$ gegeben und

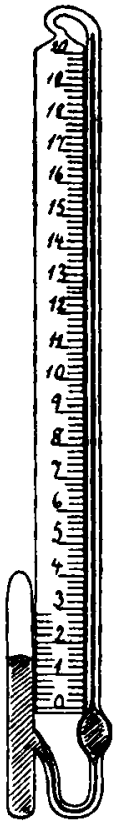

Fig. 2. vorsichtig zum Schmelzen gebracht. Allmählich wurde Eutektikum nachgefüllt, bis die flüssige Schmelze etwa drei Viertel des Zylinders ausfüllte. Die Temperatur des Bades konnte nun mit einer BunsenFlamme zwischen $300^{\circ}$ und $360^{\circ}$ sehr gut reguliert werden. Die durch Konvektionsströme ohnehin dauernd bewegte Schmelze wurde durch einen Eisendraht hin und wieder umgerührt. - Das fertig beschickte Manometer wurde mit einer kleinen Bunsen-Flamme vorsichtig erwärmt, bis das Metall geschmolzen war, und dann in das etwa $250^{\circ}$ warme Heizbad eingebängt. Zur Messung der Temperatur diente ein geeichtes Thermoelement $(\mathrm{Pt}, \mathrm{Pt} 10 \% \mathrm{Rh})$, dessen Lötstelle am oberen Ende des weiten Manometerschenkels, unmittelbar neben der Oberfläche des Amalgams lag. Bei einer Verschiebung der Lötstelle um $5 \mathrm{~cm}$ nach oben oder unten zeigte sich, daß die Temperatur in dieser Badschicht innerhalb eines Grades gleich war. Das Bad wurde nun langsam erhitzt und bei bestimmten Versuchstemperaturen so lange konstant gehalten, bis sich der Druck nicht mebr änderte. Um einem Hängenbleiben des Zinnfadens vorzubeugen, wurde vor den Ablesungen mit einem Eisendraht an die Capillare ge- 
klopft, oder der A pparat leise gescbüttelt. Starke Erschütterungen mußten vermieden werden, um ein Abreißen des Zinnfadens zu verhindern. Die Einstellungen waren im allgemeinen gut reversibel, am sichersten dann, wenn der Zinufaden sich von unten, d. h. mit steigender Temperatur einstellte. Die von oben erreichten Werte sind in Tabelle II durch einen Stern gekennzeichnet. Erbebliche Unregelmäßigkeiten traten nur bei dem verdünntesten Amalgam auf; die Werte sind ihrer Kleinheit halber ohnedies recht unsicher.

Tabelle II.

\begin{tabular}{|c|c|c|c|c|c|c|c|c|}
\hline \multicolumn{3}{|c|}{ Amalgam I $0.84 \% \mathrm{Hg}$} & \multicolumn{3}{|c|}{ Amalgam II $1.30 \% \mathrm{Hg}$} & \multicolumn{3}{|c|}{ Amalgam III $2.43 \% \mathrm{Hg}$} \\
\hline Min. & ${ }^{\circ} \mathrm{C}$ & $\mathrm{mm} \mathrm{Sn}$ & Min & ${ }^{\circ} \mathrm{C}$ & $\mathrm{mm} \mathrm{Sn}$ & Min. & ${ }^{0} \mathrm{C}$ & $\mathrm{mm} \mathrm{Sn}$ \\
\hline $\begin{array}{r}15 \\
30 \\
45 \\
63 \\
80 \\
93 \\
143 \\
158\end{array}$ & $\begin{array}{l}305 \\
325 \\
345 \\
264 \\
345 \\
366 \\
345 \\
366\end{array}$ & $\begin{array}{c}4 \\
10 \\
{[18]} \\
\overrightarrow{[18]} \\
{[31]} \\
14^{*} \\
27\end{array}$ & $\begin{array}{l}20 \\
24 \\
39 \\
48 \\
76\end{array}$ & $\begin{array}{l}305 \\
325 \\
345 \\
358 \\
366\end{array}$ & $\begin{array}{l}\left.[11]^{1}\right) \\
15 \\
20 \\
26 \\
30\end{array}$ & $\begin{array}{r}22 \\
52 \\
67 \\
77 \\
89 \\
100 \\
104\end{array}$ & $\begin{array}{l}305 \\
345 \\
325 \\
305 \\
325 \\
345 \\
325\end{array}$ & $\begin{array}{l}14 \\
37 \\
28^{*} \\
-\overline{28} \\
38 \\
28^{*}\end{array}$ \\
\hline
\end{tabular}

\begin{tabular}{|c|c|c|c|c|c|c|}
\hline \multicolumn{4}{|c|}{ Amalgam IV $4.97 \% \mathrm{Hg}$} & \multicolumn{3}{|c|}{ Amalgam V $8.00 \% \mathrm{Hg}$} \\
\hline${ }^{\circ} \mathrm{C}$ & $\mathrm{mm} \mathbf{S n}$ & ${ }^{\circ} \mathrm{C}$ & $\mathrm{mm} \mathrm{Sn}$ & Min. & ${ }^{\circ} \mathrm{C}$ & $\mathrm{mm} \mathbf{S n}$ \\
\hline $\begin{array}{l}305 \\
325 \\
345 \\
358 \\
345 \\
305\end{array}$ & $\begin{array}{l}30 \\
55(56) \\
81 \\
90 \\
81^{*} \\
32^{*}\end{array}$ & $\begin{array}{l}325 \\
345 \\
325 \\
305 \\
325 \\
305\end{array}$ & $\begin{array}{l}56 \\
79 \\
57 \\
- \\
55 \\
29\end{array}$ & $\begin{array}{r}5 \\
29 \\
40 \\
57 \\
67 \\
-\end{array}$ & $\begin{array}{l}305 \\
325 \\
345 \\
325 \\
34 . ; \\
358\end{array}$ & $\begin{array}{c}50 \\
80 \\
119 \\
78^{*} \\
118 \\
136\end{array}$ \\
\hline
\end{tabular}

Nach Abschluß jeder Versuchsreibe wurde die in der Tabelle II angegebene Konzentration des Amalgams durch eine Zinnbestimmung ermittelt. Die zur Analyse benutzte, absichtlich reichlich bemessene Probe $(3-6 \mathrm{~g})$ stammte aus der obersten Schicht des Amalgams. Die während des Versuchs dampfförmig werdenden Quecksilbermengen waren in allen Fällen so klein, daß sie außer Betracht bleiben konnten.

Wesentlich für die Richtigkeit der Messungen war die Voraussetzung, daß das Quecksilber während des Versuchs nicht in den

1) Der Wert ist zu hoch, weil ein Quecksilbertropfen in dem ausgezogenen Ende des weiten Rohres hängen geblieben war, der erst bei höherer Temperatur durch Verdampfung und Auflösung verschwand. Die eingeklammerten Werte sind weiterhin nicht benutzt worden. 
oberen Teil des Zinnfadens diffundierte. Deshalb wurde nach Beendigung jedes Versuches das obere Ende des Zinnfadens abgetrennt $(5-28 \mathrm{~mm})$ und qualitativ auf Quecksilber geprüft, indem das Metall in rauchender Salpetersäure gelöst, und in die verdünnte und von Stickoxyden befreite Lösung ein Kupferstreifen gebracht wurde. Nur in einem einzigen Falle konnte Quecksilber nachgewiesen werden, als eine Versucbsreibe ungewöhnlich lange gedauert hatte. Sie ist deshalb hier nicht aufgenommen worden.

\section{Besprechung der Resultate.}

In der Tabelle III enthalten die horizontalen Reihen die bei einer bestimmten Versuchstemperatur gemessenen Druckwerte ( $p$ in $\mathrm{mm} \mathrm{Sn}$ ) nach steigenden Konzentrationen c (in $\% \mathrm{Hg}$ ) geordnet. Tabelle IV gibt in der gleichen Weise die Quotienten p:c wieder.

Tabelle III.

\begin{tabular}{l|r|r|r|r|r|r}
\hline${ }^{\circ} \mathrm{C}$ & 0.84 & 1.30 & 2.43 & 4.97 & 8.00 & $0 \% \mathrm{Hg}$ \\
\hline 305 & 4 & {$[11]$} & 14 & 31 & 50 & \\
325 & 10 & 15 & 28 & 56 & 80 & \\
345 & 14 & 20 & 38 & 81 & 119 & $\mathrm{~mm} \mathrm{Sn}$ \\
358 & - & 25 & - & 90 & 136 &
\end{tabular}

Tabelle IV.

\begin{tabular}{c|c|c|c|c|c|c}
\hline${ }^{\circ} \mathrm{C}$ & 0.84 & 1.30 & 2.43 & 4.97 & 8.00 & $\% \% \mathrm{Hg}$ \\
\hline 305 & 4.8 & - & 5.8 & 6.2 & 6.2 & $\mathrm{p}: \mathrm{c}$ \\
325 & 11.9 & 11.5 & 11.5 & 11.3 & 10.0 & $\mathrm{p}: \mathrm{c}$ \\
345 & 16.6 & 15.4 & 15.6 & 16.3 & 14.9 & $\mathrm{p}: \mathrm{c}$ \\
358 & - & 19.2 & - & 18.1 & 17.0 & $\mathrm{p}: \mathrm{c}$
\end{tabular}

Falls das Henrysche Gesetz gültig ist, müssen die Werte von $\frac{p}{c}$ iür jede Temperatur gleich sein. Die Tabelle IV läßt erkennen, daß das nur annähernd erfüllt ist. Die vorhandenen Abweichungen sind aber so unregelmäßig um die Mittelwerte verteilt, daß sie zum größten Teil auf Versuchsfehler zurückgeführt werden dürfen. Wenn demnach die Einzelwerte auf Genauigkeit keinen Anspruch erbeben, so reicht doch ibre Zusammenstellung aus, um die gesuchte Gesetzmäßigkeit hervortreten zu lassen. Für die Auflösung von Quecksilberdampf gilt in dem Druckintervall von $4 \mathrm{~mm}$ bis $136 \mathrm{~mm} \mathrm{Sn}$ (das entspricht ${ }^{1}$ ) rund

1) Das spezifische Gewicht des flüssigen Zinns ist nach Day und Sosman bei $300^{\circ}$ : 6.943 ; bei $400^{\circ}: 6.875$. (Landolt-Börnstein, Physik.- 
$2-70 \mathrm{~mm} \mathrm{Hg}$ ) bei. Temperaturen zwischen $300^{\circ}$ und $360^{\circ}$ das Hen rysche Gesetz. Die mit dem Quarzmanometer ausgeführten Versuche (vergl. S. 1239) sprechen, wie Tabelle $V$ zeigt, dafür, daß auch für höhere Temperaturen und Drucke das Henrysche Gesetz gilt.

a)

\begin{tabular}{l|c|c|c}
\hline${ }^{\circ} \mathrm{C}$ & $2.2 \% \mathrm{Hg}$ & $10 \% \mathrm{Hg}$ & \\
\hline 515 & 173 & 770 & $\mathrm{~mm} \mathrm{Hg}$ \\
528 & 190 & 886 & $\mathrm{~m}$
\end{tabular}

Tabelle V.

b)

\begin{tabular}{c|c|c|c}
\hline${ }^{\circ} \mathrm{C}$ & $2.2 \% \mathrm{Hg}$ & $10 \% \mathrm{Hg}$ & \\
\hline 515 & 79 & 77 & $\mathrm{p}: \mathrm{c}$ \\
528 & 86 & 89 & $\mathrm{p}: \mathrm{c}$
\end{tabular}

Heycock und Neville haben die Gefrierpunkte verdünnter Lösungen von Quecksilber in Zinn bestimmt. Die molekulare Gefrierpunktserniedrigung $\mathrm{E}$ des Zinns ist nicht genau bekannt. Die in der Literatur für die Schmelzwärme angegebenen Werte schwanken zwischen 13.6 und 14.65 cal. für $\left.1 \mathrm{~g} \operatorname{Zinn}^{2}\right)$. Die neueste, von Robertson bestimmte Zahl 14.1 liegt in der Mitte. Nimmt man sie als richtig an, so ist nach der van't Hoff schen Formel:

$$
\mathbf{E}=\frac{0.02(232+273)^{2}}{14.1}=362 \text {. }
$$

Als Mittelwert aus den Gefrierpunktbestimmungen an Lösungen von 9 verschiedenen Metallen in $\mathrm{Zinn}^{3}$ ) ergibt sich aus den Messungen von Heycock und Neville: $\mathrm{E}=340^{\circ}$, unter der Voraussetzung, da $\beta$ die Metalle einatomig gelöst sind. Legt man die Zahlen für $\mathbf{E}$ zu Grunde, so berechnet sich aus den Messungen der beiden englischen Forscher ${ }^{3}$ ) das scheinbare Molekulargewicht des Quecksilbers zu 256-242. Für einatomig gelöstes Quecksilber sollte das Molekulargewicht 200 sein. $O b$ die Abweichung nach oben auf einer teilweisen Assoziation von Quecksilberatomen zu Molekülen $\left(\mathrm{Hg}_{\mathrm{g}}\right)$ beruht, oder durch die Abscheidung fester Lösungen von Quecksilber in Zinn ver-

chemische Tabellen 4. Auflage, S. 148 [1912]). Um die Drucke der Tabellen IIl und IV in $\mathrm{mm} \mathrm{Hg}$ auszudrücken, müssen sie mit $\frac{6.9}{13.6}$ multipliziert werdeb. Wir haben jede Umrechnung unterlassen, weil wir die a b sol uten Werte der Drucke nicht für so genau halten, daß sie - etwa zur Berechnung der Lösungswärme - weiter benutzt werden könnten.

1) Landolt-Börnstein, J. c. S. 829.

2) Landolt-Börnstein, l. c. S. 639; Soc. 57, 376 [1890]; vergl. auch Puschin, Z. a. Ch. 36, 201 [1903].

3) 1. c. vergl. die Zusammenstellung bei van Heteren, Z. a. Ch. 42, $150,151[1904]$. 
ursacht wird, läßt sich auf Grund der vorhandenen Angaben nicht entscheiden ${ }^{1}$ ). Immerhin wird man folgern dürfen, daß das Quecksilber in der Lösung größtenteils in einatomiger Form enthalten ist. Da auch der Quecksilberdampf einatomig ist, so muß nach dem Verteilungssatz angenähert das Henrysche Gesetz gelten. Aus der Dampfdichte des Quecksilbers und seinem Molekulargewicht in flüssigem Zinn läßt sich also die gleiche Gesetzmäßigkeit ableiten wie aus den Druckmessungen der vorliegenden Arbeit.

Die in der Einleitung angestellten Betrachtungen über die Lösungen einatomiger Gase in Metallen werden durch das untersuchte Beispiel bestätigt. Man erkennt, daß dadurch mittelbar auch die für die zweiatomigen Gase abgeleiteten Vorstellungen an Wahrscheinlichkeit gewinnen. Hätte das Experiment auch für den einatomigen Quecksilberdampf die Gültigkeit der Quadratwurzelbeziehung ergeben, so wäre dadurch die Anwendung des Verteilungssatzes auf MetallGaslösungen unzulässig geworden ${ }^{2}$ ). Das Ergebnis der vorstehenden Untersuchung aber spricht dafür, daß der Berthelot-Nernstsche Satz auch die Auflösung von Gasen in Metallen regelt ${ }^{3}$ ), und daß es eine spezifische Eigenschaft der metallischen Lösungsmittel ist, zweiatomige Gase in Atome aufzuspalten.

\section{Zusammenfassung.}

Die Dampidrucke flüssiger Zinnamalgame von $0.8-8 \%$ Quecksilber sind bei einer gegebenen Temperatur zwischen 300 und $360^{\circ}$ und für Drucke von 2-70 mm Quecksilbersäule der Konzentration des gelösten Quecksilbers annähernd proportional. Zwei einzelne Versuchsreiben mit 2 und $10 \% \mathrm{Hg}$ ergaben die gleiche Gesetzmäßigkeit für 515 und $528^{\circ}$ und ein Druckintervall ron $173-886 \mathrm{~mm} \mathrm{Hg}$. Die Auflösung des einatomigen Quecksilberdampfes in flüssigem Zinn

1) Vergl, die kritische Besprechang der vorliegenden Arbeiten über das System $\mathrm{HgSn}$ in Gũrtlers Metallographie I, 711 [1911].

2) Vergl. hierzu Z. El. Ch. 16, 712 und 713 [1910].

3) Falle, in denen der Verteilungssatz zu versagen scheint, sind bekannt. So gilt für die Lösungen von $\mathrm{SO}_{2}$ in flüssigem Kupfer die Quadratwurzel-Beziebung, ohne daß dafūr die Theorie eine Erklärung liefert. Sieverts und Bergner haben kürzlich versucht, zur Klärung dieses Widerspruches beizutragen (Ph. Ch. 82, 257 [1913], vergl. auch Schenck, und Hempelmann, Metallurgie 10, 283 [1913]). - Fūr das System Palladium-Wasserstoff gilt bei niedrigen Temperaturen die Quadratwurzed-Beziehung nicht. Der eigentūmliche Verlauf der Druckkonzentrations-Kurven wird in einer jüngst erschienenen Arbeit von Holt, Edgar und Firth (Ph. Ch. 84, 513 [1913]) auf das Zusammenwirken von Oberflächenadsorption und Absorption zurückgeführt. 
geborcht also dem Henryschen Gesetz, während bei den metallischen Lösungen $z$ weiatomiger Gase die gelöste Menge der Quadratwurzel aus dem Gasdruck proportional ist.. Das Ergebnis spricht dafür, daß die Anwendung des Verteilungssatzes auf die Lösungen von Gasen in Metallen zulässig ist, und daß bei der Absorption zweiatomiger Gase $\left(\mathrm{H}_{2}, \mathrm{O}_{3}, \mathrm{~N}_{3}\right)$ in dem Metall eine Aufspaltung der Moleküle in Atome stattfindet.

Leipzig, 25. März 1913.

\section{A. Windaus und C. Resau:}

Methyl-isohexyl-keton, ein Abbauprodukt des Cholesterins. (Zur Kenntnis des Cholesterins. XVI.)

[Aus dem Chem. Institut der Universität Freiburg i. Br.]

(Eingegangen am 3. April 1913.)

Es ist wiederholt beobachtet worden, daß beim oxydativen Abbau des Cholesterins ein wohlriechender Stoff entsteht. So schreibt Loebisch ${ }^{1}$ ), man bemerke bei der Oxydation des Cholesterins mit Kaliumbichromat und verdünnter Schwefelsäure »einen auffallenden Geruch nach den sogenannten Obstäthern, einen Geruch, der in das Destillat übergeht, wenn man die Operation in einer Retorte mit Kühler ausfübrt«. Später haben Dorée und Gardner ${ }^{2}$ ) bei ibren Studien über die Ozonisierung des Cholesterins und seiner Derivate gefunden, daß bei der Zersetzung der Ozonide mit beißem Wasser ein Geruch nach Orangenöl auftritt. Von Derivaten des Cholesterins haben sie Cholestenon, $\beta$-Cholestanol, $\beta$-Cholestanon, Koprosterin, Pseudokoprosterin, Koprostanon und die Säure $\mathrm{C}_{27} \mathrm{H}_{44} \mathrm{O}_{4}$ untersucht. Auch bei der Oxydation des Cholesterins mit Wasserstoffsuperoxyd haben sie denselben angenehmen Geruch wahrgenommen. Etwa gleichzeitig hat $\mathrm{O} . \mathrm{Diels}{ }^{3}$ ) auf dieselbe Erscheinung aufmerksam gemacht. Er schreibt darüber:

Bei der Spaltung des Cholesterinozonids mit Wasser beobachtet man »das Auftreten eines sehr charakteristischen Geruches, der etwa an den des Methyl-hexyl-ketons erinnert. $O b$ eine ähnliche Substanz aus dem Cholesterin wirklich entsteht, wage ich vorläufig auch nicht andeutungsweise zu behaupten. Ich halte aber meine Beobachtung doch für beachtenswert

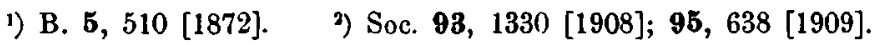

s) B. 41, 2597 [1908]. 